\title{
Toxic effects of chronic exposure to dyes among workers of synthetic textile industries
}

\author{
Ahmed F.M. Mohammed*, Magdy M. Sherif*, Ashraf I. Hasan*, Bahyeldein E. Makrahy**, Nabil E. \\ Hasan**
}

Forensic Medicine and Clinical Toxicology, Internal Medicine $(*)$ and Clinical Pathology $(* *)$ Departments, Faculty of Medicine, Al-Azhar University

Corresponding author: Ahmed F.M. Mohammed, mobile: 01009612291, email:abf114@yahoo.com

\begin{abstract}
Background: textile industry is one of the major industries in Egypt. However, the working environment is associated with potential health hazards. Aim of the work: to study the toxic effects of different types of dyes among workers in textile industries and its effect on their respiratory, renal, and immunological systems. Subjects and methods: this study was conducted on 50 male workers exposed to textile dyeing of different ages, selected from all textile plants with dyeing industry at Borg Al-Arab City compared with 50 males, who were not exposed to textile dye as a control group. Results: both groups were comparable as patient age, while smoking was significantly increased in exposed group (38.0\% vs $14.0 \%$ ). Reported symptoms such as itching, abdominal pain, etc. and respiratory symptoms were significantly increased in exposed group. There was significant increase of AST, ALT, total bilirubin and alkaline phosphatase, urea and serum creatinine in study when compared to control group. Furthermore, there was statistically significant decrease of respiratory function percentage of predicted in study group, and there was statistically significant increase of serum lead concentration in study when compared to the control group (17.18 \pm 2.35 vs $6.74 \pm 2.29$ respectively). In addition, there was significant increase of $\operatorname{IgM}$ and $\operatorname{IgG}$ in study group. Conclusion: workers in textile industry were significantly exposed to health hazards especially on respiratory system. However, multi-organ affection is the role with increased oxidative stress.
\end{abstract}

Keywords: textile, reactive oxygen species, respiratory, dyes, oxidative stress.

\section{Introduction}

Textile is one of the leading industries in the world. Approximately 60 million workers are employed in the textile industry globally ${ }^{(1)}$. The textile industry workers are exposed to a number of chemicals including dyes, solvents, optical brighteners, finishing agents and numerous types of natural and synthetic fibre dusts which affect their health. In addition, workers in the textile industry are exposed to airborne dust containing infectious, allergic and toxic substances (2). Furthermore, working conditions in the developing world are often very poor and the textile industry is not an exception to this rule. Increasing awareness of consumers through civil society campaigns has contributed to some relevant improvements, especially in large factories delivering directly to the European and American markets. However, working conditions in smaller factories that either produce for the local market or are employed by the large factories in case of higher demand are not well known, although a high proportion of the workers are employed in these small enterprises ${ }^{(3)}$.The textile manufacturing is the major industrial water user. Despite significant economic contributions, textile industries cause a range of environmental problems, mostly the pollution of water resources ${ }^{(4)}$.Textile wastewater contains various chemicals such as oil, grease, caustic soda, Glauber salt (Na2SO4), ammonia, sulfide, lead, heavy metals and other toxic substances ${ }^{(5)}$.

Typical characteristics of wastewater produced by the textile industry include high temperature, a wide range of $\mathrm{pH}$ values, biochemical oxygen demand (BOD), chemical oxygen demand (COD), total dissolved solids (TDS), heavy metals and strong pigment ${ }^{(\boldsymbol{6})}$.

\section{Aim of the work}

The aim of this work was to study the toxic effects of different types of dyes among workers in textile industries and its effect on their respiratory, renal, and immunological systems.

\section{Subjects and methods}

This study was conducted on 50 male workers exposed to textile dyeing of different ages, selected from all textile plants with dyeing industry at Borg Al-Arab City compared to 50 males, who were not exposed to textile dye as the control group. Before inclusion in this study, an informed consent was 
signed by each worker. Then, we asked them about history of exposure to the dye, time elapsed between exposure and the study and history of previous or present illness and findings were documented. In addition, respiratory symptoms were evaluated by enables questionnaire and allergic or other symptoms were evaluated. Past medical history: history of DM, hypertension, renal, liver or heart diseases, operations, blood transfusion, drug therapy and recent medications and family history of any familial disease. The clinical examination included the following: 1) General examination (vital signs, skin, eyes and characteristic odor), 2) Neurological examination, 3) Gastrointestinal system examination, 4) Cardiovascular system examination and 5) Respiratory system examination. The study was approved by the Ethics Board of Al-Azhar University.

\section{Investigations:}

Venous samples were drawn and were kept into a clean dry centrifuge tubes and left to stand for few hours before centrifugation to avoid hemolysis. Serum was separated and then used for estimation of the following: 1) Detection of toxic dye compounds in the patient serum as serum lead, 2) Biomarkers for DNA damage and oxidative stress, 3) Liver function tests and 4) Renal function tests. Blood Pb levels were determined by graphite furnace atomic absorption spectrophotometry (GFAAS) using AAnalyst 600. Serum MDA levels were measured by reacting it with thiobarbituric acid at high temperature to form pink colored complex as Kei Satoh method ${ }^{(7)}$. Nitric oxide was determined by the addition of zinc sulfate to deproteinate serum samples and then nitrate is reduced to nitrite by vanadium III and chloride followed by the addition of $100 \mu \mathrm{L}$ of Griess reagent (equal mixture of $1 \%$ sulphanilamide in $5 \%$ phosphoric acid and $0.1 \% \mathrm{~N}$-(1-naphthyl) ethylenediamine hydrochloride in distilled water). The plates were incubated for 30 minutes at room temperature and the optical density was measured at $540 \mathrm{~nm}$ using the ELISA (Enzyme-Linked Immunosorbent Assay) reader (Bio-Tek Instruments INC, USA) ${ }^{(8)}$. Suproxide dismutase (SOD) was measured with a commercially available Ransod kit (Randox Laboratories, Ltd, UK). Briefly The SOD activity was assessed by the inhibition of the reduction of nitroblue tetrazolium by superoxide anion. The assay mixture was composed of $0.05 \mathrm{M}$ phosphate buffer, $\mathrm{pH} 8.60,10.2 \mathrm{M}$ EDTA, and 10.4 M nitroblue tetrazolium; dimethyl sulfoxide were added under stirring to the above mixture. The absorbance of each solution was read at $550 \mathrm{~nm}$ using Beckman spectrophotometry (USA). One unit of enzymatic activity is defined as the amount of enzyme causing $50 \%$ inhibition of the reduction to Formosan observed in the blank ${ }^{(9)}$. Glutathione peroxidase was measured by a Ransel kit (Randox Laboratories, Ltd, UK). Its function is to catalyze the oxidation of reduced glutathione (GSH) to oxidized glutathione (GSSG) by using t-butyl hydroperoxide as the substrate for the assay of the enzyme. The rate of formation of GSSG is measured by means of the glutathione reductase reaction. The oxidation of NADPH is measured at $340 \mathrm{~nm}$ using Beckman spectrophotometry (USA) ${ }^{(10)}$. Catalase was measured by a Ransel kit (Randox Laboratories, Ltd, UK). Catalase catalyzes the breakdown of $\mathrm{H} 2 \mathrm{O} 2$ into 2 moles of water and 1 mole of oxygen. The rate of decomposition of $\mathrm{H} 2 \mathrm{O} 2$ by catalase is measured using Beckman spectrophotometry (USA)at a wavelength of $230 \mathrm{~nm}$. Ethanol is added to stabilize the hemolysate by breaking down the "complex II" of catalase and $\mathrm{H} 2 \mathrm{O} 2$ (11). Total antioxidant capacity was measured using Randox total antioxidant status kit (UK) in which ABTS (2, 2-Azinodi [3- ethyl benzthiazolin sulphanate]) is incubated with a peroxidase and $\mathrm{H} 2 \mathrm{O} 2$ to produce the radical cation ABTS+. This has a stable blue green color, which is measured at $600 \mathrm{~nm}$. Antioxidants in the added sample cause suppression of this color production to a degree which is proportional to their concentration. Immunoglobulin levels (IgM and IgG) were detected in serum samples using Abcam`s Humann kit according to manufacturer's instruction (12).

Statistical analysis of data: the collected data was organized, tabulated and statistically analyzed using statistical package for social science (SPSS) version 20 (IBM®SPSS ${ }^{\circledR}$ Inc., Chicago, USA). Qualitative (categorical) data were presented as frequency and percent distribution, while quantitative (numerical) data were presented as mean and standard deviation (SD). For comparison between groups, the independent samples ( $\mathrm{t}$ ) and Chi square tests were used for numerical and categorical variables respectively.

\section{Results}

In the present study, age was ranged from 25 to 58 years and there was no significant difference between study and the control groups (39.50 \pm 7.72 vs $41.62 \pm 7.69$ years respectively). There was significant increase of smoking in study when compared to the control group (38.0\% vs $14.0 \%$ respectively). In addition, packs/year was significantly increased in studied when compared to the control group $(13.68 \pm 5.97$ vs $7.85 \pm 3.93$ 
respectively) (Table 1). In the present work, itching, abdominal pain, constipation, malaise, muscle incoordination, depression, headache, impaired concentration, pallor and metallic taste in mouth in study group when compared to control group. In addition, there was statistically significant increase of respiratory symptoms in study when compared to control group either the total symptoms or each symptom individually (Table 2). In the present study, there was significant increase of AST, ALT, total bilirubin and alkaline phosphatase in study when compared to control group. In addition, there was significant increase of both urea and creatinine in study when compared to control group. Furthermore, there was statistically significant decrease of respiratory function percentage of predicted in study group when compared to control group. Also, there was statistically significant increase of serum lead concentration in study when compared to control group (17.18 \pm 2.35 vs $6.74 \pm 2.29$ respectively) (Table 3). In the present study, there was statistically significant increase of MDA, serum NO and significant decrease of superoxide dismutase, glutathione peroxidase, catalase and total antioxidants in study when compared to control group. In addition, there was significant increase of IgM and IgG in the study when compared to the control group (Table 4).

Table 1: patient characteristics in studied groups

\begin{tabular}{||l|l|l|l||}
\hline \hline Variable & Study group & Control group & P value \\
\hline Age & $39.50 \pm 7.72 ; \quad 25-$ & $41.62 \pm 7.69 ;$ & $25-58$ \\
& 51 & $7(14.0 \%)$ & $0.17(\mathrm{~ns})$ \\
\hline Smoking & $19(38.0 \%)$ & $7.85 \pm 3.93 ;$ & $0.006^{*}$ \\
\hline Packs/year & $13.68 \pm 5.97 ;$ & $5-15$ & $0.026^{*}$ \\
& $5-25$ & \\
\hline
\end{tabular}

Table 2: history of different symptoms other than respiratory symptoms in studied groups

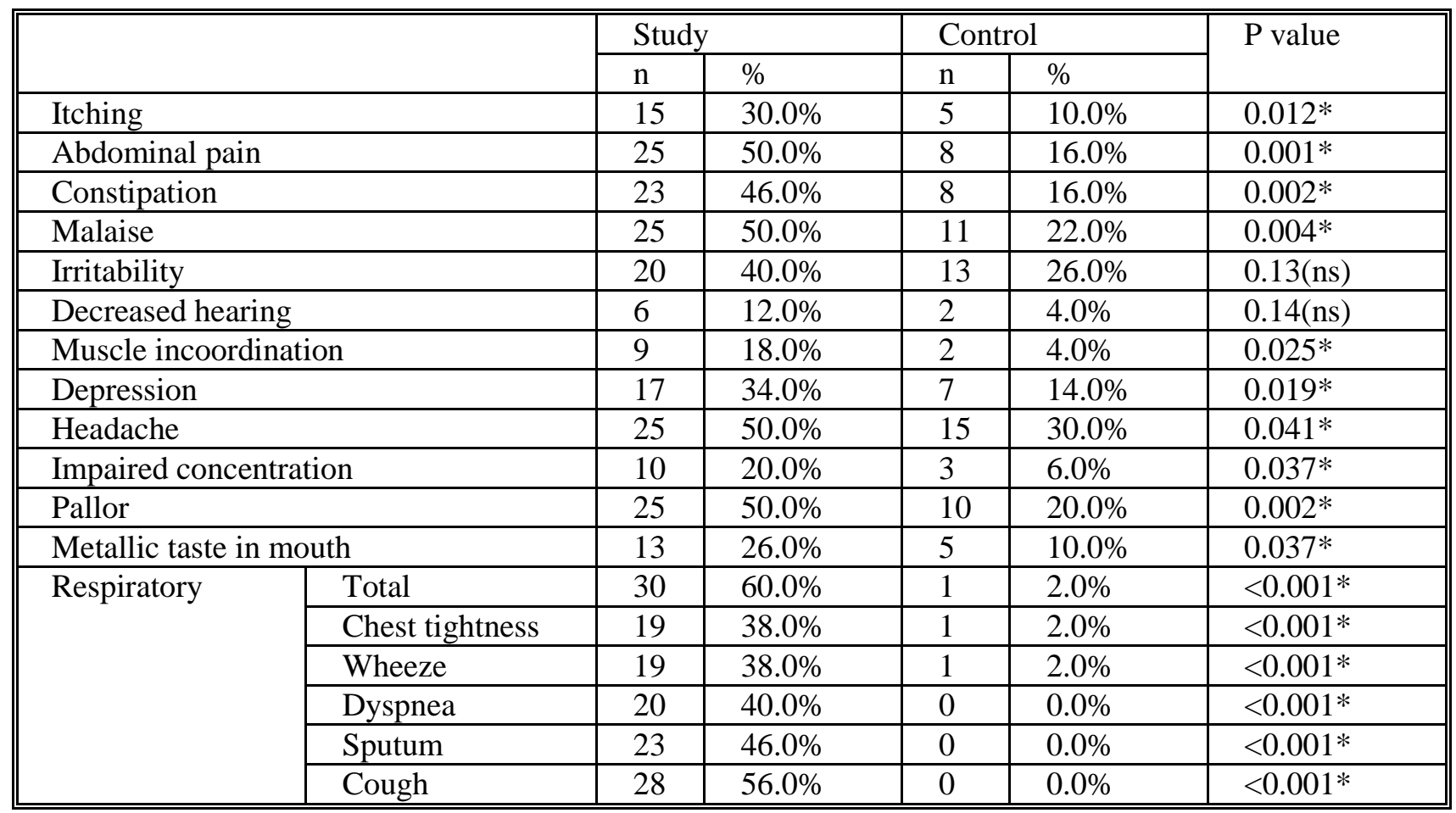

Table 3: laboratory investigations and respiratory function tests in studied groups 


\begin{tabular}{||l|l|l|l||}
\hline \hline Variable & Study group & Control group & P value \\
\hline AST & $59.04 \pm 11.99$ & $11.84 \pm 1.79$ & $<0.001^{*}$ \\
\hline ALT & $66.64 \pm 9.19$ & $12.58 \pm 1.62$ & $<0.001^{*}$ \\
\hline Total bilirubin & $1.29 \pm 0.17$ & $0.87 \pm 0.16$ & $<0.001^{*}$ \\
\hline Alkaline phosphatase & $146.32 \pm 17.26$ & $69.28 \pm 16.0$ & $<0.001^{*}$ \\
\hline Urea & $34.62 \pm 6.22$ & $18.78 \pm 3.56$ & $<0.001^{*}$ \\
\hline Creatinine & $1.29 \pm 0.19$ & $1.0 \pm 0.13$ & $<0.001^{*}$ \\
\hline FEV1\% & $76.10 \pm 4.78$ & $85.64 \pm 1.96$ & $<0.001^{*}$ \\
\hline FVC\% & $77.50 \pm 4.70$ & $86.50 \pm 2.13$ & $<0.001^{*}$ \\
\hline FEV1/FVC\% & $74.86 \pm 4.42$ & $83.74 \pm 2.50$ & $<0.001^{*}$ \\
\hline PEFR\% & $77.32 \pm 4.34$ & $84.58 \pm 2.12$ & $<0.001^{*}$ \\
\hline Lead concentration & $17.18 \pm 2.35$ & $6.74 \pm 2.29$ & $<0.001^{*}$ \\
\hline \hline
\end{tabular}

Table 4: oxidative stress markers in studied groups

\begin{tabular}{|c|c|c|c|c|c|c|}
\hline & & Mean & SD & Minimum & Maximum & P value \\
\hline \multirow[t]{2}{*}{ MDA (nmol/mL) } & Study & 6.63 & 1.19 & 3.50 & 9.50 & \multirow{2}{*}{$<0.001 *$} \\
\hline & Control & 1.90 & 0.55 & 1.00 & 2.90 & \\
\hline \multirow[t]{2}{*}{ Serum NO (umol/L) } & Study & 31.12 & 4.26 & 21.00 & 41.00 & \multirow{2}{*}{$<0.001 *$} \\
\hline & Control & 17.18 & 2.85 & 12.00 & 25.00 & \\
\hline \multirow{2}{*}{$\begin{array}{l}\text { Superoxide dismutase } \\
(\mathrm{U} / \mathrm{ml})\end{array}$} & Study & 1.39 & 0.29 & 0.80 & 2.10 & \multirow{2}{*}{$<0.001 *$} \\
\hline & Control & 4.91 & 0.54 & 3.50 & 6.10 & \\
\hline \multirow{2}{*}{$\begin{array}{l}\text { Glutathione peroxidase } \\
(\mathrm{U} / \mathrm{ml})\end{array}$} & Study & 22.94 & 3.45 & 17.00 & 29.00 & \multirow{2}{*}{$<0.001 *$} \\
\hline & Control & 50.02 & 4.27 & 41.00 & 60.00 & \\
\hline \multirow[t]{2}{*}{ Catalase (U/ml) } & Study & 182.50 & 17.82 & 145.00 & 220.00 & \multirow{2}{*}{$<0.001 *$} \\
\hline & Control & 325.72 & 33.53 & 250.00 & 392.00 & \\
\hline \multirow{2}{*}{$\begin{array}{l}\text { Total antioxidant } \\
(\mathrm{mmol} / \mathrm{L})\end{array}$} & Study & 0.74 & 0.12 & 0.36 & 0.95 & \multirow{2}{*}{$<0.001 *$} \\
\hline & Control & 2.62 & 0.26 & 2.10 & 3.30 & \\
\hline \multirow[t]{2}{*}{ IgM } & Study & 115.78 & 14.48 & 79.00 & 152.00 & \multirow{2}{*}{$<0.001 *$} \\
\hline & Control & 68.06 & 5.81 & 45.00 & 81.00 & \\
\hline \multirow[t]{2}{*}{$\mathrm{IgG}$} & Study & 1012.02 & 99.18 & 689.00 & 1350.00 & \multirow{2}{*}{$<0.001 *$} \\
\hline & Control & 671.32 & 46.62 & 580.00 & 790.00 & \\
\hline
\end{tabular}




\section{Discussion}

The present study was designed to evaluate the toxic effects of different types of dyes among workers in textile industries and its effect on their respiratory, renal, and immunological systems. Results of the present work confirmed that, workers in textile industry suffer from respiratory health hazards (as proved by frequency of respiratory symptoms and decreased respiratory functions) when compared to non-exposed workers. These results are in agreement with results Khan $\boldsymbol{e t} \boldsymbol{a l}$. $^{(13)}$ who reported a high prevalence of respiratory symptoms among workers in the cotton industry was found. The highest symptoms frequency was seen in weaving factories, especially those of small and medium size. These findings were also consistent with results of previous studies of Nafees et $\boldsymbol{a l} .{ }^{(14)}$ who reported that reported high respiratory symptoms in weaving and spinning mills. Furthermore, the results of the present work are in agreement with the study of Tageldin et al. ${ }^{(15)}$ who reported that, their study was done to observe the effects of cotton dust on respiratory symptoms and spirometric functions among cotton workers. Results revealed that, the prevalence of respiratory symptoms was higher in exposed group as compared to control group $(59.0 \%$ vs $0.0 \%)$ probably due to inhalation of cotton dust. Cough was found in $(35.0 \%)$ of workers, expectoration was found in $(22.0 \%)$ of all exposed workers so cough and expectoration were the most prevalent symptoms in this study.

Regarding respiratory functions, the results of the present work are in agreement with the results of the study done by Aminian et al. ${ }^{(16)}$ on 140 cotton textile workers and 150 office workers as control group to evaluate the effect of exposure to cotton dust on respiratory health and pulmonary function in Tehran, Iran. Their results showed that cotton workers had more respiratory symptoms as compared to control groups and greater declines were seen in forced vital capacity (FVC), forced expiratory volume in first second (FEV1) and FEV1/FVC in cotton workers. These results were compatible with the study carried out by Nagoda $e t$ al. ${ }^{(17)}$ whom found that FEV1, FVC, PEFR were significantly lower in cotton workers as compared to control.In the present study, exposed workers had significantly higher levels of liver enzymes, urea and creatinine, which reflects deleterious role of textile industry dyes on the health of those workers. Finally, oxidative stress markers were significantly higher and antioxidants were significantly lower in exposed workers and both $\operatorname{IgM}$ and $\operatorname{IgG}$ were significantly higher in exposed workers who reflect the possible inclusion of immunological system. As the major site of chemical transformation, these organs are prone to the toxic effects of chemicals. When cell membranes of the hepatocytes are damaged, a number of cytoplasmic enzymes are released into the circulatory system providing basis for clinical diagnosis. Serum AST and ALT are the most used biochemical markers of hepatocellular necrosis and are considered sensitive indicators of hepatic chemical induced injuries ${ }^{(18)}$. Significant increase in serum ALT and AST activities and concomitant increase in total and direct bilirubin concentrations in the exposed group suggest acute hepatocellular damage due to the induction of necrosis. Increase in lipid peroxidative damage induced by the toxic metals and possibly organic constituents on the hepatocytes (necrosis), increased cell membrane permeability to ALT and AST from the cytoplasm into the blood circulation. Hepatic necrosis of the liver cells and significant increase in serum MDA in the exposed group are in support of this assertion, as reported in an animal

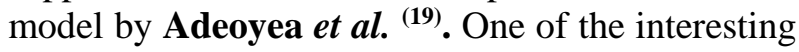
findings in the present study is the higher levels of lead in exposed workers when compared to nonexposed workers, and it may be responsible for deleterious effects observed in exposed workers. Ercal et al. ${ }^{(20)}$ reported that lead affects its target tissues by triggering reactive oxygen species/ reactive nitrogen species (ROS/RNS) generation that in turn increases lipid peroxidation, protein carbonylation, alters the balance between sulfhydryl groups (reduction in sulphydryl group is one of the important mechanisms of redox inactive metals to induce oxidative stress) and $\mathrm{Ca}^{2+}$, and promotes inflammation. Furthermore, Sun et al. ${ }^{(21)}$ reported that exposure to high level of various metals affects oxidative balance and can cause DNA damage. Lead accumulation owing to environmental exposure has been linked to oxidative DNA damage, high reactive oxygen species (ROS), oxidative stress, and depletion of catalase (CAT) and glutathione activities. All these studies support the results of the present work. Regarding oxidative stress, results of the present work are comparable to those reported by Almashhedy et al. (22) who reported that, there was significant increase of MDA as an oxidant biomarker, while the levels of vit $\mathrm{E}$ and GSH as antioxidant were decreased significantly for textile workers compared to control group. In addition, there was a significant increase of SOD and CAT activities for textile workers compared to control group.Results of the present work may be explained by suggesting that the 
various azo dyes and their products are toxigenic and mutagenic which characteristic to many aromatic amines but rather through a mechanism involving oxygen radicals and superoxide free radical was produced by the azo dyes after reduction by intestinal bacteria ${ }^{\left({ }^{23}\right)}$. On the other hand, the azo dyes metabolized into aromatic amines can generate reactive oxygen species as part of their metabolism (NOS) by interaction of these amines groups with intercellular nitrite or nitrate. The reactive oxygen species (ROS) are chemically reactive molecules containing superoxide anion, hydroxyl radical and $\mathrm{H} 2 \mathrm{O} 2$ could be produced during the metabolism of nitrosamines and increase oxidative stress (23). Under certain environmental conditions oxidative stress due to the increasing of ROS formation and decreased of the antioxidant defense mechanism of the cells including Vit.E and GSH began to consumed to prevent the cell death by these toxic radicals so their levels in the tissue homogenate were decreased specially at higher doses of ROS, on the other hand MDA level was increased as a product of lipid peroxidation occurred by the ROS action on lipids of cellular membranes. Biological membranes are particularly prone to the ROS effect, the peroxidation of unsaturated fatty acids in biological membranes leads to a decrease of membrane fluidity and disruption of membrane integrity and function, which is implicated in serious pathological changes ${ }^{(22)}$. Reactive oxygen species play an important role in pathological changes in the liver, the increasing of free radical production is able to cause auto-oxidation of the hepatic cells, resulting in marked hepatic lesions ${ }^{(24)}$. Previous studies explained the environmental pollutant can induce the oxidative stress which can be accompanied with lipid peroxidation and depleted of antioxidant defense in plant and mammalian cells ${ }^{(25)}$. Jadhav et al. ${ }^{(23)}$ explained that textile azo dyes may play a role in the generation of oxidative stress when examined the toxicity of azo dyes Remazol Red(RR) on the plants, while Phugare et al. ${ }^{(26)}$, demonstrate the formation of free radicals can lead to the lipid peroxidation reaction which are indices for oxidative stress. Nitric oxide radical has been identified as a biologically important molecule involved in a number of physiological processes, $\mathrm{NO} \cdot$ can react rapidly with superoxide anion to produce the potent oxidant peroxynitrite, which is very reactive species initiating oxidation and nitration ${ }^{(27)}$. Results of the present work are in agreement with Shaker ${ }^{(28)}$ who reported that, their results demonstrate highly significantly increased levels of oxidants biomarkers (serum MDA and
NO.) in exposed group when compared to controls. Increased lipid peroxidation in the blood of exposed subjects warns that oxygen free radicals have increased in the body and thus might attack cells, which, in the long-term, results in multi-organ damage. They added, serum glutathione peroxidase (GPX) which is a selenoenzyme responsible for elimination of ROS, was significantly lowered in cotton exposed workers in comparison to their controls ( $23.75 \pm 6.27,50.56 \pm 8.90$, respectively, $\mathrm{P}$ $<0.05)$. Decrease in the activity of antioxidants may be due to exhaustion of the enzymes by reactive oxygen species ${ }^{(\mathbf{2 9})}$. Our results are also in agreement with that of Suryakar et al. ${ }^{(30)}$ who reported highly significant increases in serum MDA and nitric oxide in cotton industry workers as compared to controls. The activities of SOD, glutathione peroxidase, glutathione reductase were found to be significantly decreased in cotton industry workers. Their study demonstrated significant decreases in catalase activity and total antioxidant status in exposed workers. They concluded that cotton dust exposure induces oxidative stress among textile industry workers which may contribute to respiratory disorders.In the present work, we measured IgM and $\mathrm{IgG}$ as markers of the humoral immunity. The results showed statistically significant higher levels of immunoglobulin $\mathrm{M}$ and immunoglobulin $\mathrm{G}$ in exposed workers compared to control group. Our results are in agreement with those obtained by Sultana et al. ${ }^{(31)}$ who found that occupational exposure to cotton dust causes a wide range of physical and psychological health problems in the garment workers that may also affect their immune function as evident by the significant increase in the mean IgG level in cotton exposed workers when compared to the controls. They conclude in their research that garment workers constantly inhale fiber particles which can probably stimulate antibody production (including $\mathrm{IgG}$ ); these antibodies could kill the bacterial cells.

In summary, workers exposed to dust and different dyes in textile industry were under great oxidative stress as manifested by a rise in oxidant biomarkers and a reduction in antioxidants. In addition, dust stimulates humoral immunity as detected by the increases in the immunoglobulin levels. Furthermore, respiratory symptoms were increased and function was decreased. These bio-functional markers and simple respiratory function tests might be useful in screening and surveillance for occupational hazard.

\section{References}

1. Forstater M (2009): Implications of the 
global financial and economic crisis on the textile and clothing sector. Geneva: International Labour Organization. International Labour Office, 5: 15-44.

2. Singh $\mathbf{Z}$ and Chadha $\mathbf{P}$ (2016): Textile industry and occupational cancer. Journal of Occupational Medicine and Toxicology, 11:39-46.

3. Khan AW and Nersesyan A (2014): Bioeffects monitoring of workers in the cotton industry. Biomonitoring, 1:39-45.

4. Ahmed A and Tareq SM (2008): Textile industries in Bangladesh: a rising environmental degradation down the drains. Bangladesh Textile Today. Available at: 〈http:// www. textiletoday .com. bd/textile-industries-in-bangladesha-rising-environmental degradation/.

5. Islam M, Chowdhury M, Billah $M$ et al. (2012): Investigation of effluent quality discharged from the textile industry of Purbani group, Gazipur, Bangladesh and its management. Bangladesh J. Environ. Sci., 23: $123-130$.

6. Nabil SK, Azad A and Khan MS (2014): Flow segregation options to minimize pollution load and optimize ETP option. In: Proceedings of the $5^{\text {th }}$ International Conference on Chemical Engineering (ICChE).http://che.buet.ac.bd/4thinternational-conference-on-chemicalengineering-icche-2014/

7. Satoh K (1978): Serum lipid peroxide in cerebro-vascular disorder determined by a new colorimetric method. Clin. Chim. Acta., 90: 37-43.

8. Ghasemi A, Hedayati $M$, Biabani $H$ (2007): Protien precipitation methods evaluated for determination of serum nitric oxide end products by the Griess assay. Journal of Medical Science Research, 2:2932.

9. Arthur JR and Boyne $R$ (1985): Superoxide dismutase and glutathione peroxidase activities in neutrophils from selenium deficient and copper deficient cattle. Life Sci., 36: 1569-1575.

10. Paglia DE and Valentine WN (1967): Studies on the quantitative and qualitative characterization of erythrocyte glutathione peroxidase. J. Lab. Clin. Med., 70: 158-69.

11. Aebi H (1973): Catalases. In: Bergmeyer HU (Ed.) Methods of Enzymatic Analysis. Academic Press, New York. pp: 73-75. http://dx.doi.org/10.1016/b978-0-12-
091302-2.50032-3

12. Miller N, Rice-Evans C, Davies MJ et al. (1993): A novel method for measuring antioxidant capacity and its application for monitoring the antioxidant status in premature neonates. Clin. Sci., 84:407-412.

13. Khan AW, Moshammer $H$ and Kundi $M$ (2015): Industrial hygiene, occupational safety and respiratory symptoms in the Pakistani cotton industry. BMJ. Open, 5:7275.

14. Nafees AA, Fatmi $Z$ and Kadir MM (2013): Pattern and predictors for respiratory illnesses and symptoms and lung function among textile workers in Karachi, Pakistan. Occup. Environ. Med., 70:99-107.

15. Tageldin MA, Gomaa AA and Hegazy EA (2017): Respiratory symptoms and pulmonary function among cotton textile workers at Misr Company for Spinning and Weaving EL-Mahalla, Egypt. Egyptian Journal of Chest Diseases and Tuberculosis, 66: 369-376.

16. Aminian $O$, Amir $S$ and Sadeghniiat $K$ (2013): Study of respiratory symptoms and pulmonary function in cotton textile workers. J. Basic Appl. Sci. Res., 3 (4): 33 36.

17. Nagoda M, Okpapi JU and Babashani M (2012): Assessment of respiratory function among textile workers at kano textile mills, kano, Nigeria. Niger. J. Clin. Pract., 15 (4): 373-379.

18. Timbrel JA (2009): Principles of Biochemical Toxicology, $4^{\text {th }}$ edition, New York, USA, pp:13-41.

19. Adeoyea GO, Alimbab CG and Oyelekeca OB (2015): The genotoxicity and systemic toxicity of a pharmaceutical effluent in Wistar rats may involve oxidative stress. Toxicology Reports, 2: 1265-1272.

20. Ercal N, Gurer-Orhan $H$ and AykinBurns N (2001): Toxic metals and oxidative stress Part I: mechanisms involved in metal-induced oxidative damage. Curr. Top. Med. Chem., 1: 529539.

21. Sun L, Gao Y, Zhang et al. (2014): Effect of high fluoride and high fat onserum lipid levels and oxidative stress in rabbits. Environ. Toxicol. Pharmacol., 38: 10001006.

22. Almashhedy LA (2013): Toxicity 
assessment of textile dyes via oxidative stress hypothesis for iraqi textile workers. Int. J. Pharm. Bio. Sci., 4(4): 577 - 587.

23. Jadhav SB, Phugare SS, Patil PS et al. (2011): Biochemical degradation pathway of textile dye Remazol red and subsequent toxicological evaluation by cytotoxicity, genotoxicity and oxidative stress studies International. Biodeterioration and Biodegradation, 65: 733-743.

24. Valavanidis A, Vlahoganni T, Dassenakis M et al. (2006): Molecular biomarkers of oxidative stress in aquatic organisms in relation to toxic environmental pollutants. Ecotoxicol. Environ. Saf., 64(2): 178-89.

25. Jeng HA, Pang CH, Iwara $\mathrm{N}$ et al. (2011): Polycyclic aromatic hydrocarbon-induced oxidative stress and lipid peroxidation in relation to immunological alteration. Occupational and Environmental Medicine, 68(9):653-658.
26. Phugare SS, Kalyani DC, Patil AV et al. (2011): Textile dye degradation by bacterial consortium and subsequent toxicological analysis of dye and dye metabolites using cytotoxicity, genotoxicity and oxidative stress studies. Journal of Hazardous Materials, 186:713-723.

27. Bartesaghi S, Trujillo M, Denicola A et al. (2004): Reactions of desferrioxamine with peroxynitrite-derived carbonate and nitrogen dioxide radicals. Free Radic. Biol. and Med., 36(4): 471-483.

28. Shaker DA (2012): Oxidative stress and immunoglobulin levels in workers exposed to cotton dust. Egyptian Journal of Occupational Medicine, 36(1): 107-121.

29. Perricone $\mathrm{C}$, De Carolis $\mathrm{C}$ and Perricone R (2009): Glutathione: a key player in autoimmunity. Autoimmun. Rev., 8: 697701.

30. Suryakar AN, Katkam RV and Dhadke VN (2010): A study of oxidative stress in cotton industry workers from Solapur city. Biomedical Research, 21(3): 260-264

31. Sultana R, Ferdous KJ, Hossain M et al. (2012): Immune functions of the garment workers. Int. J. Occup. Environ. Med., 3(4):195-200. 